\title{
Design, Implementation and Evaluation of Audio for a Location Aware Augmented Reality Game
}

\author{
Natasa Paterson Katsiaryna Naliuka Soren Kristian Jensen Tara Carrigy Mads Haahr \\ Fionnuala Conway \\ Trinity College Dublin \\ College Green \\ Dublin 2, Ireland \\ natasa.paterson,katsiaryna.naliuka,soren.jensen,tara.carrigy@ndrc.ie \\ mads.haahr@cs.tcd.ie,conwayfi@tcd.ie
}

\begin{abstract}
In this paper, the development and implementation of a rich sound design, reminiscent of console gaming for a location aware game, Viking Ghost Hunt (VGH) is presented. The role of audio was assessed with particular attention to the effect on immersion and emotional engagement. Because immersion also involves the interaction and the creation of presence (the feeling of being in a particular place) these aspects of the sound design were also investigated. Evaluation of the game was undertaken over a three-day period with the participation of 19 subjects. The results gained imply that audio plays an important role in immersing a player within the game space and in emotionally engaging with the virtual world. However, challenges in regards to GPS inaccuracy and unpredictability remain, as well as device processor constraints, in order to create an accurate audio sound field and for the real-time rendering of audio files.
\end{abstract}

\section{Categories and Subject Descriptors}

C.5.3 [Computer System Implementation]: Microcomputers; H.1.2 [Models and Principles]: User/Machine Systems; H.5.1 [Information Interfaces and Presentation]: Multimedia Information Systems; H.5.2 User Interfaces; H.5.5 Sound and Music Computing.

\section{General Terms}

Design, Experimentation, Human Factors.

\section{Keywords}

Sound Design, Location Aware Gaming, Immersion, Engagement.

Permission to make digital or hard copies of all or part of this work for personal or classroom use is granted without fee provided that copies are not made or distributed for profit or commercial advantage and that copies bear this notice and the full citation on the first page. To copy otherwise, or republish, to post on servers or to redistribute to lists, requires prior specific permission and/or a fee.

Fun and Games 2010, September 15-17, 2010, Leuven, Belgium.

Copyright 2010 ACM 978-1-60558-907-7/10/09...\$10.00.

\section{INTRODUCTION}

In the last 35 years, game audio has evolved considerably by moving away from the dependence on midi based wavetable synthesis bleeps, beeps and simplistic melodies to three dimensional (3D) sound effects, orchestral soundtracks and believable dialogue. Console gaming has more processing power available to synthesise sounds and real-time complex sound effects, 3D localisation and reverberation - especially in a 5.1 surround sound configuration. Hence there is a trend to replace a part of recorded audio by real time generated sounds and music. Therefore, for a composer and sound designer there are many more avenues available in creating a complex and immersive soundscape. Such game console examples are Silent Hill [21] and Thief [24] which present engaging sound designs and an interactive use of audio.

Immersive gaming soundscapes have mainly been associated with a static console set up but with the introduction of Personal Digital Assistants (PDAs) capable of greater processing power than their predecessors, gaming audio can now move away from the dependability on midi wavetable synthesis sound to include richer soundscapes. An example of this is Soul Trapper [22], which uses spatialisation and a complex audio backdrop to convey narrative elements of an audio adventure game with some visual interaction. However, most mobile phone based games still pay minimal attention to the overall audio design, which are mainly interested on conveying information and appropriate user interface sounds.

The increased use of GPS (Global Positioning System) enabled smartphones, have allowed developers to create real world location aware gaming where the virtual world can be overlaid onto the physical world. As audio has developed for console applications, the challenge is now to incorporate some of these features in a location aware scenario on the mobile platform that is not only for navigational purposes but that is immersive, reactive to the gamers' movements and that adds to the sense of presence. Developing a realistic and ambitious sound design on the limited mobile phone CPU (Central Processing Unit) creates challenges and requires innovative approaches and ideas. The emotional and immersive impact of complex sound designs in location gaming has as yet, not been thoroughly explored.

The aim of this research is to develop a sound design with a complex and varied soundscape that is immersive, emotionally engaging and supportive of the game narrative. The goal is to implement this on the mobile platform with all the technical 
constraints that mobile gaming can bring. In location aware gaming, visual displays are often small therefore other sensory modalities such as sound can be investigated in regards to player game experience. As well as the implementation of an augmented reality game, the sound design will be assessed for its ability to convey the desired soundscape environment relative to the game context, the development of player immersion and emotional engagement. First we shall take a brief look at the background of location aware gaming on the mobile platform, then describe Viking Ghost Hunt as our working prototype and the sound design and subsequent implementation. The user trials undertaken examined the usability of the game interface and the overall game experience, which included audio elements. Specifically the immersive quality of the audio and how this added to the game experience was investigated. This testing process will be described together with the results obtained and their significance. Finally we conclude on the possible role of audio in location aware gaming and directions for further research.

\section{RELATED BACKGROUND}

There has been increasing interest in location aware gaming since the availability of PDAs that incorporate integrated GPS capabilities. This integration allows for the overlay of a digital mediascape onto the physical world that responds to contextual cues such as GPS. Location aware gaming layers a virtual world onto the physical world in such a way that physical elements of the gamers real world can be integrated within the game structure thereby merging the virtual and physical worlds [5] which in turn can produce a sense of a place (a space is a physical environment and a place has greater meaning) [25]. The sound design of a location aware game can convey important game information such as navigational sounds and instructional dialogue thereby enabling the user to look away from the visual graphical interface and to remain engaged within their physical environment. Listening to audio requires less cognitive attention than visual information and therefore reduces the amount of player distraction from the game, hence encouraging the player to remain in the game space [5]. Research has already shown that the use of a realistic sound design can help build excitement and tension in the game world and an enhanced sense of immersion, without the use of visual graphics [26]. To facilitate user engagement within the virtual world, a sense of presence (the perception of being in a particular space) must be created which is interrelated and dependant on player emotional engagement and immersion. Hence a multisensory approach of using both visual and audio interfaces is increasingly being implemented for enhancing immersion and it has been found that audio is a significant element for multimodal applications such as location aware gaming [11].

Investigations into using non-speech audio messages (earcons) ${ }^{1}$ concluded that audio cues were significant for conveying information and for the development of semantic meaning [12]. This is important as it suggests that audio can provide useful information about virtual environments and support perceptual integration. In regards to location aware applications, most audio investigation has focused on non-gaming applications, artistic installations or navigational tools for the visually impaired. Research into spatialised sound in a location aware application

\footnotetext{
${ }^{1}$ Sounds used to convey information about computer systems.
}

was undertaken by Dr. Kirsten Cater and Dr. Richard Hull et al [3], with initial results indicating that the soundscape of a PDA application can help users navigate in a physical environment by conveying relevant information through audio. In their application, a 2-axis compass, Bluetooth GPS and HP iPAQ was used. Sound installations such as 'The Tactical Sound Garden' [23] explore the concept of overlaying soundscapes onto a specific urban space. In this example, wireless (WiFi) 'hot zones' were utilised to install a 'sound garden' for public use which used an element of audio for navigation and spatialisation. Demor [7] is a location aware $3 \mathrm{D}$ audio first person shooter game primarily designed for the blind but which sighted players can also take part. This game investigated psychoacoustic properties for the presentation of a 3D soundscape that would be accurately presented and used by players for real physical space navigation. In this example, a laptop, GPS tracker and headphones with a head-tracking device together with a joystick is used. These applications mainly focus on sound as navigation and not on potential immersive or experiential properties. Additionally, the applications were not based on the mobile platform and often involved complex technical set-ups.

An example of location aware audio in gaming is 'The Songs of North' [8]. This application investigates the use of audio for navigation and in the conveying of game information with the research containing some reference to the possible role of immersion in the game experience. This location aware mixed reality game features audio as an important aspect, however it must be noted that headphones were not used in this example. The application was also hampered by challenging technical constraints in the presentation of an audio soundscape due to memory and processor limitations.

There are many more examples of the use of audio in location aware gaming, however most research has focused on audio as a function of navigation and in the conveying of game information rather than its emotional and immersive effect. There is currently research on console game audio [9], however game audio mobility and its implications has not been thoroughly dealt with.

\section{GAME PROTOTYPE}

Viking Ghost Hunt (VGH) is implemented on the Android platform using the HTC T-Mobile G1 and is a working prototype for a location aware game in Dublin city centre, Ireland. The game is narrative driven and played around the old Viking sites of Dublin with the player acting as a paranormal investigator. The gamer uses various visual and audio interfaces together with stereo headphones to locate and interact with the ghosts. The $V G H$ aim is to immerse the player in the game world which is merged with the physical world, with the PDA acting as a paranormal investigative device. The aim of the audio design was to create a soundscape that is both informative of the game environment and that is immersive and emotionally engaging. Sound also supports the contextual element of $V G H$ to create a believable space and to blur the real and virtual world borders. The aim of the game is to locate paranormal activity and gather evidence that manifests visually or aurally. This evidence may give information about the ghostly activity and clues for moving through the game space. The paranormal device (mobile phone) has different modes or interfaces that can be used to locate paranormal manifestations in the real world space and to capture audio-visual evidence. The modes of paranormal investigation consist of camera/x-ray mode, 


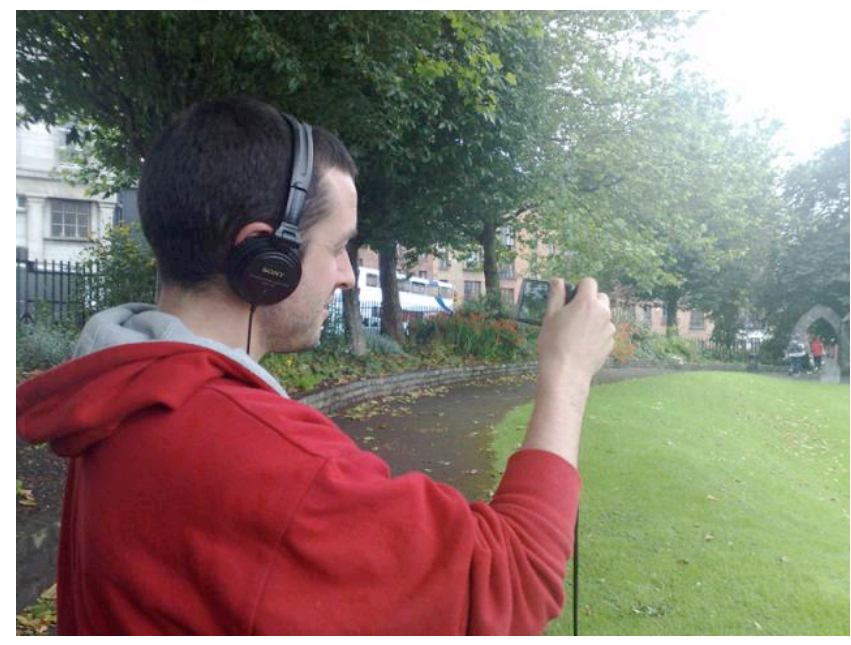

Figure 1. Viking Ghost Hunt prototype

map mode, radar mode and frequency scanner mode. The frequency scanner mode is an audio interface reminiscent of Electromagnetic Voice Phenomenon (EVP) often used by paranormal investigators. Paranormal investigators believe that 'ghostly voices' can be heard when radio static is analysed. Gamers must use the frequency scanner in order to find the correct ghost frequency (Hertz), record the white noise audio and subsequently hear the decoded ghostly message on playback. This is an integral part in the game mechanics for player interaction, game information, evidence retrieval and in the delivery of the game narrative.

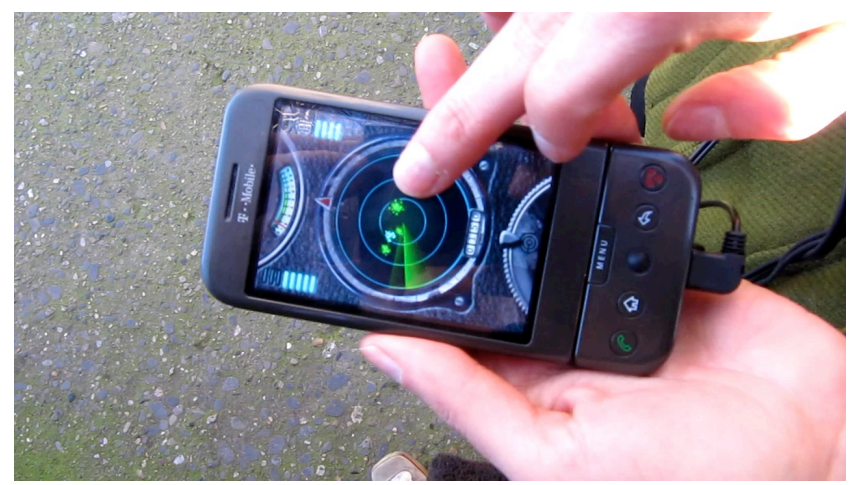

Figure 2. Radar mode

In addition to the frequency scanner, audio is also used for all of the user interface sounds in order to support the concept that the mobile phone is a paranormal investigative device. Automated ambient audio and sound effects are triggered when in proximity to GPS locations or regions to assist the player in navigating to the correct locations and for immersion and engagement in the game experience.

\section{SOUND DESIGN}

The aim of the sound design is to create an immersive experience in which the player remains engaged with their physical environment. Therefore a balance between the ghostly atmosphere of the game and the location environment was maintained. Hence, sounds used are representative of both the location environment and game atmosphere. The sound samples used for $V G H$ were stereo field recordings, sourced samples or created electronically using a midi synthesizer and audio sequencer. The required sounds were placed into four categories: paranormal, environmental, musical and user interface sounds. The table below shows some of the sounds used.

Table 1. Sound Categories

\begin{tabular}{|c|c|c|c|}
\hline Paranormal & Environmental & $\begin{array}{l}\text { Musical } \\
\text { Elements }\end{array}$ & User Interface \\
\hline $\begin{array}{l}\text { footsteps } \\
\text { floorboards } \\
\text { creaking } \\
\text { bangs } \\
\text { high pitch } \\
\text { metallic } \\
\text { squeal } \\
\text { breathing } \\
\text { screams } \\
\text { whispers } \\
\text { faraway } \\
\text { voices } \\
\text { growls } \\
\text { scratching } \\
\text { moaning } \\
\text { modulated } \\
\text { voices } \\
\text { rattling } \\
\text { chains } \\
\text { battle sounds } \\
\text { ghost } \\
\text { dialogue }\end{array}$ & $\begin{array}{l}\text { animal sounds } \\
\text { church bells } \\
\text { laughing } \\
\text { children playing } \\
\text { school bell } \\
\text { paper flying } \\
\text { around } \\
\text { thunder } \\
\text { wind } \\
\text { rain } \\
\text { traffic } \\
\text { dog barking } \\
\text { chimes } \\
\text { birds } \\
\text { leaves } \\
\text { wind in trees } \\
\text { people talking } \\
\text { fire }\end{array}$ & $\begin{array}{l}\text { minor scales } \\
\text { chromatics } \\
\text { pitched and } \\
\text { unpitched } \\
\text { drones } \\
\text { drums } \\
\text { ghostly pan- } \\
\text { flute } \\
\text { augmented } \\
\text { intervals } \\
\text { tremolos } \\
\text { irregular } \\
\text { rhythms }\end{array}$ & $\begin{array}{l}\text { old AM radio } \\
\text { sounds } \\
\text { white noise } \\
\text { audio static and } \\
\text { interference } \\
\text { metal detector } \\
\text { sounds } \\
\text { dial clicks } \\
\text { Geiger metre } \\
\text { clicks } \\
\text { button sounds } \\
\text { sonar sound } \\
\text { compass sounds } \\
\text { transitional } \\
\text { sound }\end{array}$ \\
\hline
\end{tabular}

From a design perspective, audio is used similarly as in the genre of film and divided into background sound, sound effects and dialogue with the addition of user interface elements. The background consists of the creation of an atmospheric soundscape that represents the game space and the physical location of the given narrative. This background sound sets the mood of the location and virtual game space by using randomised, overlapping looped audio files in order to avoid habituation and hence player disinterest. Sound effects are then added that are relevant to the location and game narrative together with appropriate dialogue, which can be accessed using the frequency scanner mode.

The sound is triggered by creating physical zones in the form of concentric rings organised around a GPS location with varying radial distances. This enables different sound files to be triggered due to ongoing GPS update information provided by player movements. For example, as the player comes within a paranormal zone a looped drone sound (rumbling, low frequency) and some environmental sounds (e.g. wind) begin. The mixing of environmental and game sound supports the blending of the virtual and real world to such an extent that the player may believe some sounds to be part of the physical locations. As the player continues to move towards the central point of activity, sound files will start that are related to the paranormal entity. 
These sounds are descriptive of the game narrative and historical location of the game and consist of short sound effects (e.g. chains etc) that may be randomly triggered or looped. Hence players can obtain information related to the game space without looking at the mobile device which enables them to remain engaged in the physical space.

Table 2. Example of sound design for physical locations

\begin{tabular}{|c|c|c|c|c|}
\hline Location & Ghosts & $\begin{array}{c}\text { Intended } \\
\text { Mood }\end{array}$ & $\begin{array}{l}\text { Background } \\
\text { Sound }\end{array}$ & $\begin{array}{l}\text { Sound } \\
\text { Effects }\end{array}$ \\
\hline $\begin{array}{l}\text { Stairway to } \\
\text { Hell } \\
\text { (stairway } \\
\text { going down } \\
\text { into a tunnel) }\end{array}$ & Leper & $\begin{array}{l}\text { Fearful, } \\
\text { dark, } \\
\text { uncertain }\end{array}$ & $\begin{array}{l}\text { Low } \\
\text { frequency } \\
\text { rumbling } \\
\text { drone using } \\
\text { randomised } \\
\text { overlapping } \\
\text { minor } \\
\text { intervals }\end{array}$ & $\begin{array}{l}\text { Chains, } \\
\text { moaning } \\
\text { Shallow } \\
\text { breathing } \\
\text { Fire } \\
\text { sounds } \\
\text { Footsteps }\end{array}$ \\
\hline $\begin{array}{l}\text { Schoolhouse } \\
\text { Lane } \\
\text { (laneway } \\
\text { next to } \\
\text { medieval } \\
\text { church) }\end{array}$ & $\begin{array}{l}\text { Ghostly } \\
\text { children }\end{array}$ & $\begin{array}{l}\text { Eerie, } \\
\text { open } \\
\text { space }\end{array}$ & $\begin{array}{l}\text { Wind with } \\
\text { infrequent } \\
\text { low damped } \\
\text { percussive } \\
\text { booms }\end{array}$ & $\begin{array}{l}\text { Children } \\
\text { playing } \\
\text { in the } \\
\text { distance } \\
\text { School } \\
\text { bells } \\
\text { whispers }\end{array}$ \\
\hline
\end{tabular}

As the player reaches the GPS defined location, an escalation of sounds due to increasing amplitude and sound file additions signals to the player that they are entering a paranormal zone. The visual interface indicates whether the paranormal manifestation is visual or aural and the player must choose the appropriate mode to gather the evidence. As the player leaves the location after obtaining the evidence, these sounds gradually dissipate in intensity and fade away. Therefore different sounds enter and remain for different lengths of time depending on the location of the player in relation to the GPS point, hence providing an interactive, immersive experience. The ambient audio soundscape is always triggered automatically by player proximity to physical game locations.

Within the sound design the audio can also be used for navigational support by providing verbal instructions in the frequency scanner mode dialogue and by triggering sounds near paranormal location that increase in volume and complexity as the player moves closer to the location. This navigational strategy also includes the addition of various sound effects related to the paranormal activity that are triggered as the player moves toward the physical location, again informing the player that they are entering a paranormal zone. Constant background sound between paranormal locations is avoided so as not to overload the user experience and take away from the immersive experience.

In order to design and implement a complex and engaging soundscape, properties of sound must be explored and integrated in order to develop an engaging and realistic audio design. A somewhat 'natural' audio presentation that reflects real world sound has been found to be more immersive and engaging for augmented reality applications [14]. The psychoacoustics of sound in the real world in relation to spatialisation and reverberation is an important factor in creating the sound design for $V G H$ as well as the creative composition. Sound is presented in a three dimensional manner (3D) in the environment and human survival depends on the ability to establish a sense of spatial orientation. This is needed for localisation, distance perception and recognition [1] of objects, and spatialisation in an artificial soundscape can mimic how sound is heard in the real world. In order to perceive from where a sound source is originating, the human brain uses information derived from the interaction of the sound wave with that of the torso, shoulders, head and ears. Interaural time differences (ITD) and interaural level differences (ILD) of sound as it interacts with the head help to localise sound in space. For example, if the sound were located to the right, the right ear would hear the sound first and then the left with a slight time delay (ITD). The amplitude (volume) level would also be greater on the right ear than the left (ILD) and therefore the sound is perceived to originate from the right [10]. ITD is used to differentiate the location of lower frequencies while ILD is used to differentiate the location of higher frequencies. Perceptually humans use a combination of these systems, however ITD and ILD do not work as well for sound originating on the azimuth axis (vertical plane) and therefore can be poor for front and back sound localisation. For a true 3D representation of audio the use of Head Related Transfer Functions (HRTFs) must be implemented to give a realistic spatialisation of sound as it takes into account the accurate individual interaction of the user's head with the sound wave [11]. By using HRTFs individually a player sometimes cannot tell virtually spatialised sound from the real thing.

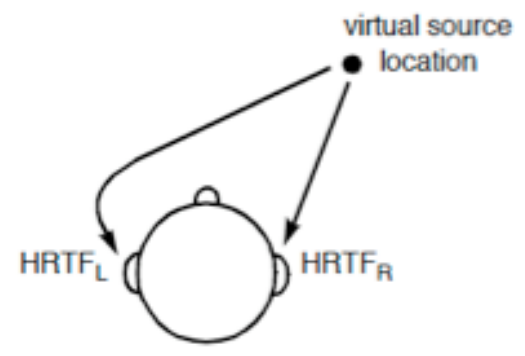

Figure 3. ILD, ITD and HTRF [14]

However the use of HRTFs is not realistic for a mobile phone (PDA) application as it requires real-time processing of audio filters which is CPU intensive and unachievable at this level for PDAs at this time. Therefore in order to spatialise sound for the $V G H$ prototype, a stereo representation of sound was found to be adequate with the use of ILD and ITD parameters in either a prerendered format or by real-time live processing of audio files, CPU permitting. For the pre-rendered formats, sound files were spatialised using a binaural simulator (software designed to imitate realistic spatialisation) or panned by changing right and left volume levels (ILD) in relation to the virtually placed source. Therefore sounds such as whispers or dialogue can be perceived as if to be moving around and in and away from the player.

Spatialised audio can be integral to navigate through a virtual world and create realism and facilitate immersion. Immersion is an important aspect of a location aware game as the user's full attention can be captured by the experience and research as shown that spatialised sound can increase immersion [14]. 
Reverberation is another important psychoacoustic consideration when developing the sound design. Reverberation is sound that is reflected from physical surfaces and back into the environment. Numerous 'early' reflections are then themselves reflected and form an 'ambient' sound field [18] and these sound reflections have been found to contribute to the sense of a space and the perception of a sound source size and its distance [2]. The addition of realistic artificial reverberation in the sound design of a location aware game can add a sense of realism and envelopment (sense of immersivity in a reverberant sound field) for the user. Hence the use of reverberation was an important stylistic element in creating a ghostly atmosphere as research as found that reverberant ambient sound can add to immersion and player engagement [14]. In the prototype, audio files were processed using different types of reverberation settings which were dependant on the context of the game locations.

In order to create a dense and engaging game atmosphere, the sound design involved the playback of a complex configuration of multiple and varied simultaneous audio files that were created with keeping in mind psychoacoustic principles, the blending of the physical and virtual worlds and the game narrative. Hence the sound design was delivered with the use of closed supra-aural headphones (to allow some external environmental sound to be heard) without the use of a head-tracking device. Therefore it was suggested to players that they face the direction of the device to ensure proper delivery of spatialised audio events.

\section{EVALUATION}

\subsection{Method of evaluating the sound design}

Objective findings such as physiological changes are difficult to measure for emotional response and immersivity to sound and music [17] as it does not take into account cognitive aspects. Therefore for a phenomenological experience (experienced from the first-person point of view), subjective reports were used for the assessments. The location aware prototype, $V G H$ was evaluated over a period of three days. The 19 participants were each provided with a G1 HTC mobile phone and headphones. Participant demographics included male and female volunteers with ages ranging from 20 years to 45 years with a mixture of previous game experience to little or no game experience. Volunteers were not briefed regarding the importance and evaluation goals of the sound design. Game feedback focused on the prototype user interface usability and game experience and took the form of a questionnaire, which included certain questions focusing on the effect and role of the sound design for emotional response and immersion.

The questionnaire consisted of open-ended questions (e.g. "Did you feel more or less engaged at different stages of the game? Which part of the game was immersive and why?" and "Which features of the interface most helped guide you to the places where the ghosts were located? Please describe) which were designed specifically not to lead the volunteers to comment on the audio unless it had a significant impact on the game experience. It also consisted of a set of statements in which 9 were specifically related to audio with players responding by using a 5-item scale (strongly disagree to strongly agree). Audio statements included: "The sound made the game feel scary", "I feel that the sound was reactive to my movements" and "The audio was seamless and felt a real part of the game".

\subsection{Results of the game audio experience}

Generally, the overall game experience was well received with $70 \%$ of participants responding that they enjoyed the game.

An important question in regards to sound was in participant expectation of the role of audio in augmented reality gaming. It was found that the respondents expected the role of audio to be roughly equal in the form of narrative and sound effects.

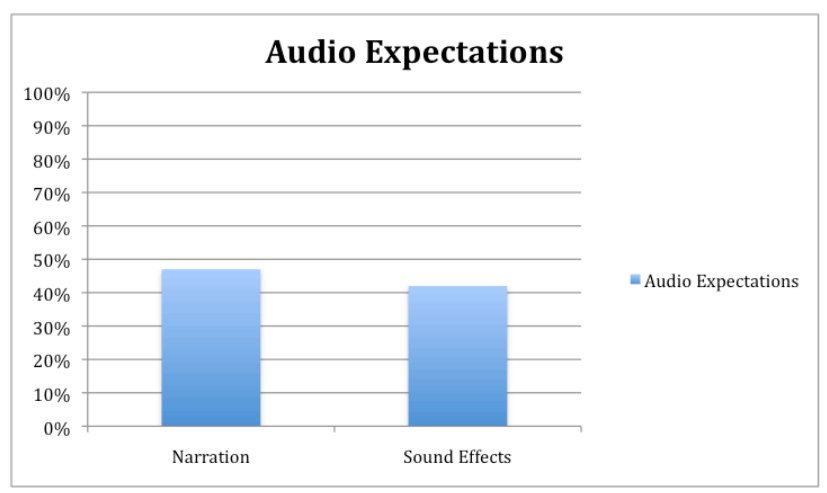

Figure 4. Audio expectations in augmented reality gaming

Overall, the participants responded positively (71\%) to the sound design, with comments such as:

"Addition of audio greatly increased the atmosphere and engagement."

"I felt immersed in the game"

"It was a very immersive experience. A couple of times I found myself not realising that I am on a street and there are people around me."

"At the moment it seems like the atmosphere is the unifying element (also one of the strongest aspects of the game)."

This positive feedback regarding the sound design was an encouraging outcome.

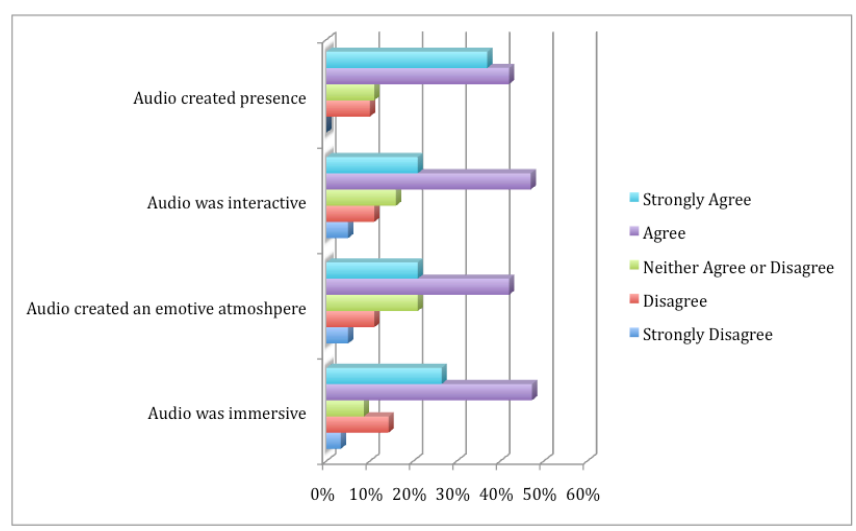

Figure 5. Summary of audio feedback

The overall response to the role of audio for game immersion was high with $70 \%$ of players agreeing or strongly agreeing to the immersion statements. By creating an engaging and complex soundscape and dialogue taking into account psychoacoustic 
considerations, participants felt involved in the game and in their role as a paranormal investigator. This engagement and immersion adds to the possible development of an emotional response felt or perceived within a game space and a sense of game presence. When asked whether the game audio contributed to the game feeling scary, $63 \%$ of participants responded in agreement or strong agreement that the "sound effects created a scary atmosphere."

Additionally, as well as investigating audio for immersion and emotional engagement, user interface usability was also investigated. For audio, this included an element of interactivity between the player, physical locations and the device user interface. As discussed previously, audio reactivity to player movement was achieved by volume changes and the addition and deletion of sound files triggered by varying distances from given GPS locations, together with the implementation of realistic paranormal investigative device sounds. Congruency between audio changes and player movement can support player involvement and therefore immersion, as the device and player actions are perceived to be coupled and realistic. In multi-modal presentations, congruency is an important factor in reinforcing a natural mode of interaction [27]. That is, the game world should reflect the experiences of real world interactions which increases the likelihood that there is a wiling suspension of belief and hence immersion [4]. Participants were asked whether they found the sound reactive to the physical environment and if they thought the user interface sounds complemented the visual interfaces and paranormal device sounds. In response, $84 \%$ of testers agreed or strongly agreed that the user interface sounds supported the roleplay element of the game play and $68 \%$ felt that the game sound was reactive - which supports the blending of the virtual world and physical one:

"The backing audio changing as I moved location was a good touch".

"Loved the sound - that was new for mobile gaming - location sensitive."

Additionally, $79 \%$ of testers felt that the audio supported the game environment in the physical locations.

In creating a complex sound design with many layers and types of sounds playing simultaneously, care must be taken in the presentation of the audio files. This was evident in some of the feedback as sound effects were at times found to overpower the narrative dialogue, which is essential to the game play. Hence a balance must be struck between important audio game information and ambient background or sound effects:

"Felt a little scary, however the first time the ghost spoke I had difficulty understanding what he said over the sound effects. Though the sound was quite good."

This brings to light another challenge for location-based audio external noise and busy environments. Even though in this example most testers did not feel distracted or interrupted by external noise this might have been due to the fact that a quiet location was sourced as a testing ground. Therefore augmented reality game locations must be carefully sourced:

"Stopping in the middle of a path was distracting as I felt I was in the way [of people]....isolated paths and lanes were more atmospheric and I felt more immersed."
Generally the results obtained from the user trials were extremely positive in regards to the sound design aim of trying to emulate console gaming audio and to be immersive and potentially emotive. As one tester noted:

"The sound - that was new and reminded me of Silent Hill on PS2 (brilliant because of sound)."

Therefore mobile phone gaming in a location aware application has the potential to be immersive and engaging even when faced with the challenges of slower processing speeds and limited memory space when compared to its static console counterpart.

\section{DISCUSSION}

In designing, implementing and testing the ambitious soundscape design for Viking Ghost Hunt, it was noticed that there are two main challenges when developing a location aware game that impacted on all aspects of the prototype - GPS inaccuracy, unreliability and mobile device technical constraints. During the user trials, GPS updates at certain times either did not occur or were inaccurate in determining the player location. This produced graphical user interface anomalies and resulted in audio files not being triggered at the intended locations. It was obviously a distraction from the game play and affected enjoyment and immersion.

The other main issue for audio in augmented reality applications is the processing speed of the device and the memory available. In the Viking Ghost Hunt prototype, the game application is stored on the mobiles' internal memory with the game assets residing on the SD (Secure Digital Memory Card) card. Very little processing power is available for any real-time audio manipulation. In order for audio to be truly reactive and to accurately represent sound spatially, live processing of sound in regards to GPS and compass positions in a physical location, must be possible. An example of this presentation is Demor [7], which uses real-time processing of spatialised sound to create a truly 3D audio virtual space. Players can accurately hear sounds emanating from the left and right in relation to their head positions and physical location. Players can also judge if a sound is located at a distance or placed close by. In this application, audio is reactive to the players' GPS locations with audio files being adjusted accordingly by live processing on a specially written audio-engine. The audio engine designed for Demor [7] would most likely have used a non-individualised HRTF (Head Related Transfer Function) database for the 3D representation, involving large amounts of data processing. Simplified psychoacoustical considerations have been implemented in the $V G H$ prototype however this has centered on including most of the spatialised sound in a pre-rendered format. Some location reactive left and right audio panning has been designed and implemented that is able to represent virtual sound in a static physical location by using GPS co-ordinates and internal phone compass sensors. However, the location and direction sensitive audio panning is currently device dependant and has not been fully tested. Player immersion and engagement could be improved in location aware gaming with a more accurate presentation of audio on the mobile platform that included a noninvasive head-tracking compass.

Careful consideration must also be taken when choosing locations for location aware applications. Such issues as noise levels, environmental player distractions and safety must be taken into account. Certain locations would be more immersive than others 
and combing this with narrative relevance would require thorough investigation before game development and implementation. Safety for gamers when wearing headphones can be an issue, especially if the audio is particularly immersive as high levels of audio immersion may take away from the intended physical location creating a sense of disengagement and pose problems in regards to traffic or unwanted attention.

Creating a sound design that intends to bring the sonic richness of console gaming into location aware applications requires the consideration of many factors outside the realm of audio. The combination of technical aspects, location, game narrative and mechanics all add to the game experience. Sound design is not simply a process of developing impressive quality audio samples but instead requires a sensitive compositional approach, as excellent sound quality does not always equate to immersion.

\section{CONCLUSIONS AND FUTURE WORK}

In this paper we have presented the sound design, implementation and evaluation of our working prototype Viking Ghost Hunt. The design demonstrated that complex audio similar to that of popular console games can to some extent, be implemented on a mobile device, even with the challenging technological constraints. This research highlights the importance of audio in location aware gaming for immersion and engagement, and hence for the overall game experience. Audio in location aware gaming is an area still not thoroughly investigated in regards to emotional impact and immersiveness and the role that this could potentially play in this genre. Also, research has not delved into the area of representing audio in a psychoacoustically correct way that would increase perception of sound naturalness and hence increase player engagement. Further research into these areas together with a possible subjective and objective investigation of player emotional and physiological reactions could be undertaken.

Location aware games can provide the user with more understanding and knowledge of their environment through both the audio and visual mediums. It is the hope of this investigation to present the importance of sound for immersion and engagement and that it may inform future applications for this increasingly popular genre.

\section{ACKNOWLEDGMENTS}

We'd like to thank the rest of the $V G H$ team; Alan Duggan, Daniel Crowley, Tina Hedayet and Roisin Cotton for their input and knowledge in the development and evaluation of the location aware prototype. We'd also like to thank the National Digital Research Centre in Dublin Ireland, for their continued generous support of the project.

\section{REFERENCES}

[1] Ashmead, D.H., Hill, E.W., \& Talor, C.R. Obstacle perception by congenitally blind children. Perception and Psychophysics, 46(5) (1989), 425-433.

[2] Begault, D. 3D Sound for Virtual Reality and Multimedia. Academic Press Inc, 1994.
[3] Cater, K., Hull, R., Melamed, E., Hutchings, R. An Investigation into the use of Spatialised Sound in Locative Games. CHI 2007, April 28 - May 3, San Jose, USA.

[4] Coleridge, S. T. The Collected Works of Samuel Taylor Coleridge: Biographia Literaria, edited by James Engell and W Jackson Bate, Princeton university Press, Princeton, New Jersey, USA, 1983.

[5] Dede, C. Immersive interfaces for engagement and learning. Science, 323 (5910) (2009), 66-69.

[6] De Souza e Silva, A. Location Based Games: Blurring the borders between physical and virtual spaces, ISEA (2004).

[7] Demor - www.student-kmt.hku.nl/ g7/redirect

[8] Ekman, I., Ermi, L., Jussi, L., Nummela, J., Lankoski, P., Mäyrä, F. Designing Sound for a Pervasive Mobile Game, ACE 2005

[9] Collins, K. Game Sound: An Introduction to the History, Theory and Practice of Video Game Music and Sound Design. MIT Press, USA, 2008.

[10] Gardner, W.G. Spatial Audio Reproduction: Toward Individualized Binaural Sound. The Bridge, V 34 (2004).

[11] Gardner, W.G., \& Martin. K. D. HRTF measurements of a KEMAR. Journal of the Acoustical Society of America, 97 (6) (1995), 3907 - 3908.

[12] Gaver, W. W., Smith, R. B., O’Shea, T. Effective Sounds in Complex Systems: The Arkola Simulation. Proceedings of the SIGCHI Conference on Human Factors in computing systems, p. $85-90,1991$.

[13] Hendrix, C. \& Barfield, W. The sense of presence within auditory virtual environments. Presence: Teleoperators and Virtual Environments, 5 (1996), 274- 289.

[14] Hollier, M. P., Rimell, A. N., Burraston, D. Spatial audio technology for telepresence. BT Technology Journal, Vol 15 (1997) No 4

[15] Keller, P. \& Stevens, C. Meaning from environmental sounds: Types of Signal-Referent Relations and their effect of recognizing Auditory Icons. Journal of Experimental Psychology: Applied, 10, 1 (2004), 3-12.

[16] Maynes, C. Worldizing - take studio recordings to the field and make them sound organic. The Editors Guild Magazine Vol. 25 (2004), No.2.

[17] Meyer, L., Emotion and Meaning in Music. University of Chicago Press, Chicago, USA, 1956.

[18] Rumsey, F. Spatial Audio. Focal Press, Oxford, UK, 2001.

[19] Sanchez, J., \& Lumbreras, M. 3D aural interactive hyperstories for blind children. International Journal of Virtual Reality, 4(1) (1998), 20-28.

[20] Sheridan T.B. Musings on Telepresence and Virtual Presence. Presence: Teleoperators and Virtual Environments, 1 (1) (1992), 120-125.

[21] Silent Hill www.konami.com/games/shsm

[22] Soul Trapper http://www.rtaudioadventures.com/Soultrapper.html

[23] Tactical Sound Garden http://www.tacticalsoundgarden.net/

[24] Thief www.gamethief.com 
[25] Nisi, V., Oakley, I., Haahr, M. Location-Aware Multimedia Stories: Turning Spaces into Places. ARTECH 2008.

[26] Valente, L., Sieckenius de Souza, C., Feijo, B. An exploratory study on non-visual mobile phone interfaces for games. IHC 2008.
[27] Witmer, B.G., Singer, M.J., Measuring Presence in Virtual Environments: A Presence Questionnaire. Presence, Vol. 7 (1998), No. 3, 225-240. 